\title{
Weaving animal temperament into food webs: implications for biodiversity
}

\author{
Nicholas P. Moran, Bob B. M. Wong and Ross M. Thompson \\ N. P. Moran (http://orcid.org/0000-0002-7331-0400) (nicholaspatrickmoran@gmail.com) and B. B. M. Wong, School of Biological Science, \\ Wellington Rd, Clayton, Victoria 3800, Australia. - R. M. Thompson, Inst. for Applied Ecology, Univ. of Canberra, Bruce, ACT, Australia.
}

\begin{abstract}
Recent studies into community level dynamics are revealing processes and patterns that underpin the biodiversity and complexity of natural ecosystems. Theoretical food webs have suggested that species-rich and highly complex communities are inherently unstable, but incorporating certain characteristics of empirical communities, such as allometric body size scaling and non-random interaction distributions, have been shown to enhance stability and facilitate species coexistence. Incorporating individual level traits and variability into food web theory is seen as a future pathway for this research and our growing knowledge of individual behaviours, in the form of temperament (or personality) traits, can inform the direction of this research. Temperament traits are consistent differences in behaviour between individuals, which are repeatable across time and/or across ecological contexts, such as aggressive or boldness behaviours that commonly differ between individuals of the same species. These traits, under the framework of behavioural reaction norms, show both individual consistency as well as contextual and phenotypic plasticity. This is likely to contribute significantly to the effects of individual trait variability and adaptive trophic behaviour on the structure and dynamics of food webs, which are apparently stabilizing. Exploring the role of temperament in the context of community ecology is a unique opportunity for cross-pollination between ecological fields, and can provide new insights into community stability and biodiversity.
\end{abstract}

\section{The biodiversity paradox}

Despite almost a century of ecological research, the persistence of highly biodiverse communities through time remains a paradox. The 'biodiversity paradox' revolves around the fact that there are many more species present in natural communities than are predicted by the principles of niche occupancy and competitive exclusion (Clark et al. 2007). Simple models of food webs predict many fewer species than are in fact observed in nature (McCann 2000). While classical ecological theory would suggest that subtle differences must exist between species to permit their coexistence, more recent theory suggests that 'ecological equivalence' of species can occur (Hubbell 2006). An understanding of processes which underpin species coexistence and persistence has wide ranging applications in ecology and environmental management, from managing invasive species (Baiser et al. 2010, Ho et al. 2011) to predicting the effects of climate change (Binzer et al. 2012).

Food-web analysis measures the flow of energy through communities and commonly shows that natural communities are both biodiverse and characterised by non-random structures, such as triangular motifs of one consumer and two resources (Milo et al. 2002). There is a stark contrast between this and the landmark community modelling work of May (1972), which showed a negative relationship between community complexity and stability in simple models of randomly interacting species. Stability refers to the tendency of a system to return to equilibrium following a perturbation, and the persistence of large, complex and species-rich communities is paradoxical based on our current understanding of ecological processes (Allesina and Tang 2012).

The strength, distribution and diversity of interactions (e.g. predator-prey, competitive and mutualistic) across communities of organisms are closely related to its stability (Gross et al. 2009, Allesina and Tang 2012). Simplistic models of food webs assume that a species interacts uniformly with its prey and predator species, ignoring that individuals or groups of individuals may specialize on certain resources, or differ in their susceptibility to predation (Valdovinos et al. 2010). Incorporating intraspecific variability in trophic roles influences food webs through multiple mechanisms, such as altering the strength of interactions between species, with intraspecific variability decreasing average interaction strength and increasing the average number of links between each node/species within the food web (Bolnick et al. 2011). These have stabilising effects on the dynamics of food webs (Dunne et al. 2002, Gross et al. 2009). Broadly, researchers are now developing a more nuanced understanding of which characteristics of organisms, species and their interactions may promote and maintain biodiversity. For example, the inclusion of 'real world' ecological 
characteristics, such as allometric body-size scaling (Brose et al. 2006, Tang et al. 2014), diversity and heterogeneity of interaction types (Akihiko and Michio 2014, Wenfeng and Kazuhiro 2014) and adaptive foraging behaviour (Uchida et al. 2007, Valdovinos et al. 2010), has improved our understanding of the processes which support biodiversity. These characteristics are commonly found to have a positive effect on stability in complex food webs, often by reducing the strength of predator-prey and competitive interactions, which are thought to have destabilizing effects (Allesina and Tang 2012).

The study of individual differences in animal behaviour, via temperament traits, may be a factor that influences the dynamics of communities, and in particular their stability. Temperament (or personality; Wolf and Weissing 2012) traits are consistent differences in behaviour between individuals of the same species, which are repeatable across time and/or across ecological contexts (Réale et al. 2007). Researchers are increasingly recognising the importance of individual traits and intraspecific variability in ecology, from both the community (Thompson et al. 2012b) and behavioural ecology perspectives (Sih et al. 2012, Wolf and Weissing 2012). For example, trait differences between individuals, such as temperament, influence the outcome of ecological interactions that make up food webs (Bolnick et al. 2011). In addition, there is a complex relationship between temperament and adaptive trophic behaviour (ATB), i.e. the ability of animals to behaviourally respond to changes in their trophic environment to increase fitness, which has also been shown to affect community stability (Valdovinos et al. 2010). A growing body of experimental research is demonstrating that responses to ecological pressures linked to temperament may be common, via ontogenic effects on the behavioural type of individuals (McGhee and Travis 2011, Niemela et al. 2012, Adamo et al. 2013, McDermott et al. 2014) and temperament-biased selection (Réale and Festa-Bianchet 2003, Bell and Sih 2007). Here, we will describe the growing body of evidence that temperament traits influence trophic interactions, and suggest approaches to temperament and community studies that will allow greater integration of these currently disparate areas of ecology.

\section{Resource segregation within species - the role of temperament}

A simple mechanism through which variation in temperament may influence stability is through resource segregation at a population level. Variation in temperament is related to differences in resource use among individuals (Kobler et al. 2009, Patrick and Weimerskirch 2014), suggesting temperament promotes resource segregation. Total niche width, i.e. the variance in the niche occupied by a species, is divided into within-individual and between-individual components (Montiglio et al. 2013). Total niche width being equal, populations with high between-individual niche variance will have higher degrees of individual resource segregation, whereas high within-individual niche variance suggests greater overlap of resource use between individuals. The portfolio effect is one mechanism in population dynamics through which temperament's effects on resource segregation may enhance population stability, where decreased competition between individuals with high phenotypic differences buffers populations against temporal fluctuations (Bolnick et al. 2011).

Empirical evidence suggests that resource segregation can be a response to increased intraspecific competition (Araujo et al. 2011) and specialisation facilitates resource partitioning

\section{Glossary}

Community. All organisms that are present within an ecosystem, which may be defined spatially and temporally, for example an aquatic community may be defined to include all aquatic organisms that are found within a lake over a year.

Food web. This includes all the organisms within a community and the links/trophic interactions between those species.

Trophic interaction. This refers to the consumer-resource or predator-prey interactions that make up a food web, i.e. one organism consuming another both within and between species.

Interaction strength. In a food web context, this is a measure of the degree that one species affects another species' population size, biomass or production, generally in a predator-prey interaction (Thompson et al. 2012a).

Connectance. The proportion of all possible links within a food web that occur, as a measure of how interconnected a food web actually is (Thompson et al. 2012a).

Omnivory. Omnivory occurs where a species within a food web feeds across multiple trophic levels, which will positively affect connectance (Thompson et al. 2012b).

Stability. Stability of food webs refers to the tendency for the food web to return to its previous structural configuration following a perturbation (Allesina and Tang 2012).

Persistence. Persistence in model food webs is measured as the proportion of species remaining following a simulation, to measure the likelihood of extinctions arising from certain food-web models (Stouffer and Bascompte 2011).

Robustness. The minimum proportion of species that, if removed from a food web will induce a significant collapse (generally $>50 \%$ species loss; Dunne et al. 2002).

Temperament. Temperament (or personality; Wolf and Weissing 2012) traits are consistent differences in behaviour between individuals of the same species, which are repeatable across time and/or across ecological contexts (Réale et al. 2007). 
and lower levels of intraspecific competition (Ravigne et al. 2009). Svanbäck and Bolnick (2007) showed that populations of three-spine sticklebacks Gasterosteus aculeatus in environments with increased intraspecific competition had higher levels of between-individual diet variance. Individuals with differing morphological traits specialized on specific prey items, demonstrating that competition can drive resource segregation - and that intraspecific trait differences are a mechanism through which this can occur within ecological time scales.

The links between temperament and resource use suggest that behavioural differences positively influence population-level persistence. Intraspecific differences in metabolic requirements and food intake appear to be associated with temperament to a degree (Careau et al. 2008, Bell et al. 2009). Temperament has been linked to differing prey selection in largemouth bass Micropterus salmoides, where less exploratory individuals preferentially fed on more novel prey types (Nannini et al. 2012). Temperament traits, particularly boldness differences, have been linked to differences in where an animal forages. A study of Steller's jays Cyanocitta stelleri showed that bolder individuals fed on a wider range of food sources (Rockwell et al. 2012). In blackbrowed albatross Thalassarche melanophrys, the boldness of individuals was associated with spatial variability in foraging, where shyer individuals would forage further from their colony than bold individuals (Patrick and Weimerskirch 2014). Also, temperament can determine an individual's role within social structures (Bergmüller and Taborsky 2010), which can be seen as a mechanism to assist resource allocation within populations. For example, between-individual differences in the behaviour of social spiders Stegodyphus spp. are increased by repeated social interactions (Laskowski and Pruitt 2014, Modlmeier et al. 2014a). By facilitating social structures, variation in temperament may promote resource allocation, mitigate intraspecific competition and positively influence population-level persistence (Montiglio et al. 2013).

Plastic changes in temperament traits in response to environmental factors can mitigate the negative effects of intense intraspecific competition. For example, aggressive behaviours can facilitate the establishment of dominance relationships within social structures, allowing allocation of resources without conflict (Ang and Manica 2010). High aggression can be one potential context-specific plastic response to conflict and increased competitive pressure (Wilson et al. 2011), but studies looking at aggression as a consistent temperament trait have shown similar patterns. In bluefin killifish Lucania goodie, food resource limitation in the early life stages produced adult males with more aggressive temperaments (McGhee and Travis 2011), which may be linked to social resource allocation in adults. Similarly, in great tits Parus major, resource limitation in early life stages was shown to increase aggression and exploration traits in some individuals (Carere et al. 2005). Resource segregation may be enabled by the presence of temperament traits in populations and by changes in the behavioural type of individuals. Furthermore, diet differences have been shown to promote the development of intraspecific trait variation, both morphological and behavioural (Oudman et al. 2016). As resource segregation can increase the stability and potential size of a population, it is a simple mechanism that could promote the development of individual behavioural differences within populations. This is possible where variation in temperament facilitates more stable population structures, giving it a systemic selective advantage (Borrelli et al. 2015). This further suggests that temperament will influence a community's stability by increasing the persistence of its constituent species.

\section{Linking animal temperament and trophic interactions}

A growing body of experimental research is showing that an animal's temperament is associated with ecological interactions, particularly those relevant to community dynamics (Table 1, Fig. 1). Community dynamics have generally been studied using network analysis tools, focusing on trophic, competitive and mutualistic interactions (Dunne 2006, Allesina et al. 2008, Wenfeng and Kazuhiro 2014). Trophic interactions (i.e. one organism consuming another) have received the majority of attention and are particularly important to the complexity-stability relationship in food webs (Allesina and Tang 2012). Here, we look at the connection between trophic interactions and temperament from both the consumer's perspective (i.e. feeding and foraging) and the prey's perspective (i.e. predation effects). In addition, we consider connections between temperament and competitive interactions, which are interactions that negatively influence the complexity-stability relationship in communities (Allesina and Tang 2012).

\section{Predator-prey interactions: the consumer's perspective}

An animal's temperament can be linked to its individual diet and foraging method, and the collective foraging behaviours of a population are also influenced by temperament traits (Michelena et al. 2010). In multiple species of birds, individual's behavioural type determined the role (producer $\mathrm{v}$ scrounger) they took within a foraging group (Kurvers et al. 2012b, Jolles et al. 2013). Similarly, the composition of behavioural types within a group influences the outcome of collective decision making. This can influence a group's choice of where to forage (Michelena et al. 2010), foraging methods (Keiser and Pruitt 2014), foraging success (Kurvers et al. 2012a, Blight et al. 2016), as well as collective defence behaviours (Modlmeier et al. 2014b). Temperament traits in predators appear to be a major source of intraspecific variability influencing predator-prey interactions, and changes to the composition of behavioural types in populations is a potential mechanism through which group foraging can adapt to change.

There is strong evidence that temperament traits are associated with between-individual differences in adaptive foraging behaviours, i.e. the ability to switch between foraging methods and food sources. This is shown in the relationship between certain traits and the temporal variability and predictability of food sources. In guppies Poecilia reticulata, individuals raised on an unreliable, temporally variable food source show increased boldness levels as adults (Chapman et al. 2010). Furthermore, bolder individuals can show different foraging preferences based on their reliability, with shy individuals preferring more predictable food sources 


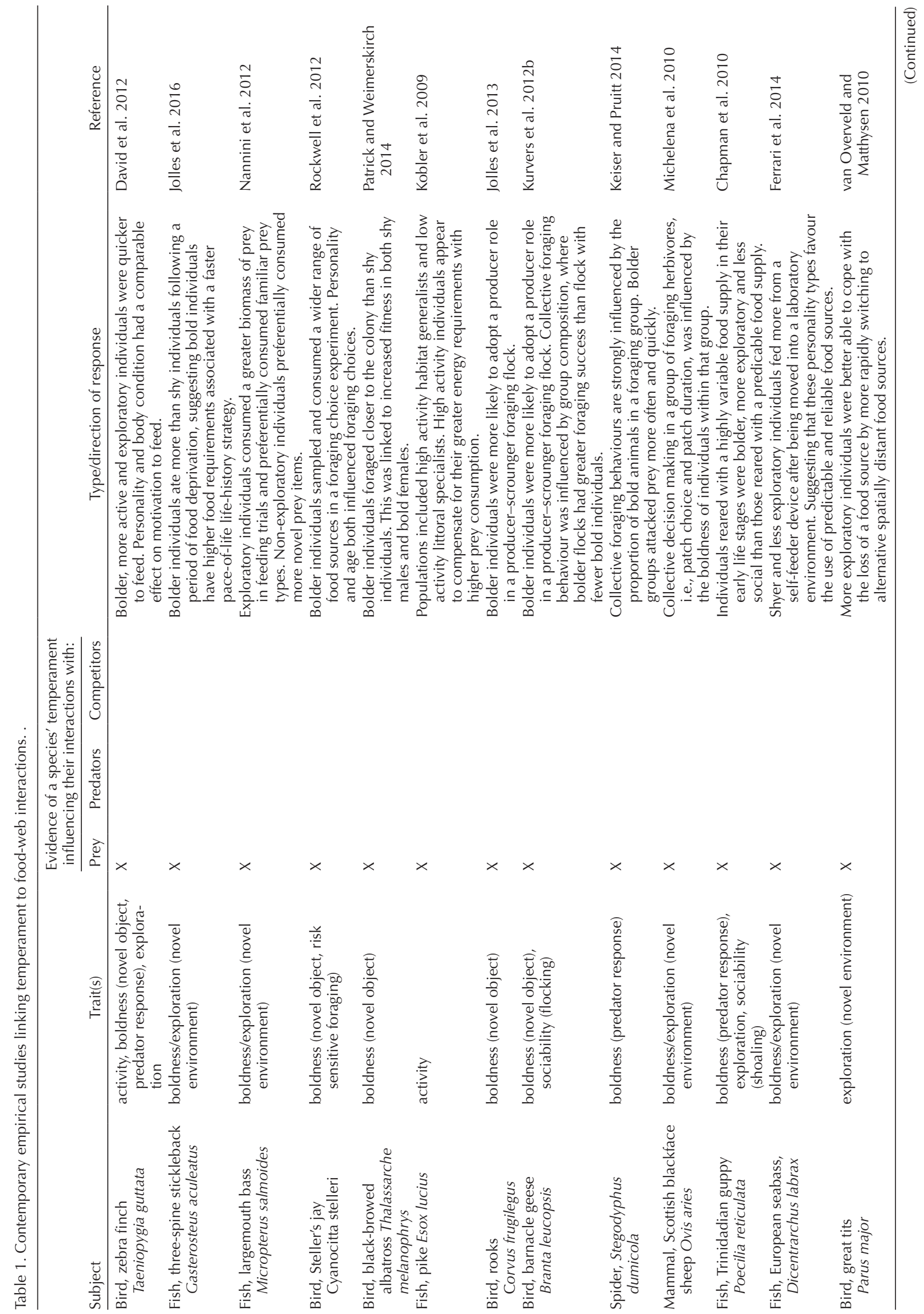




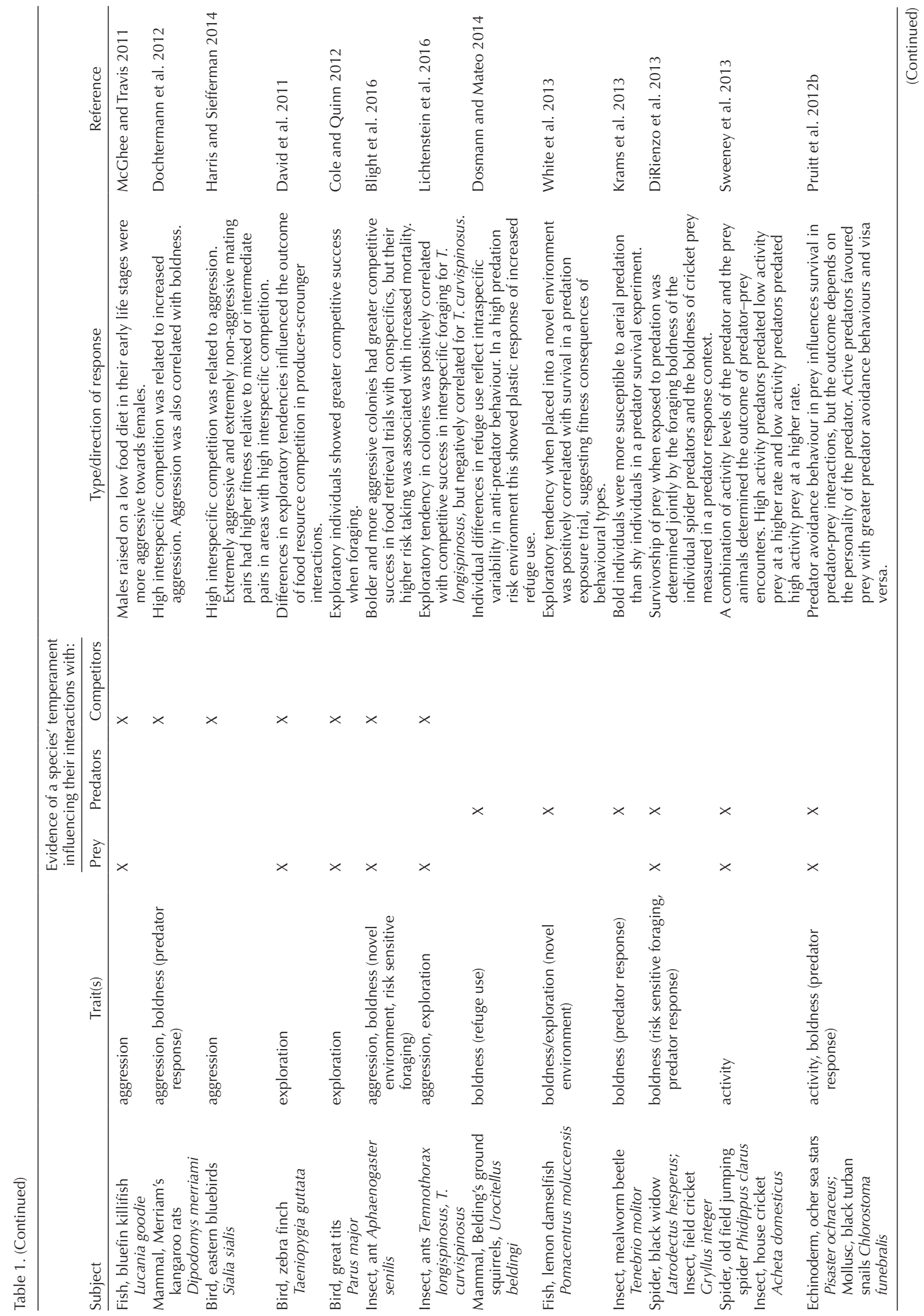




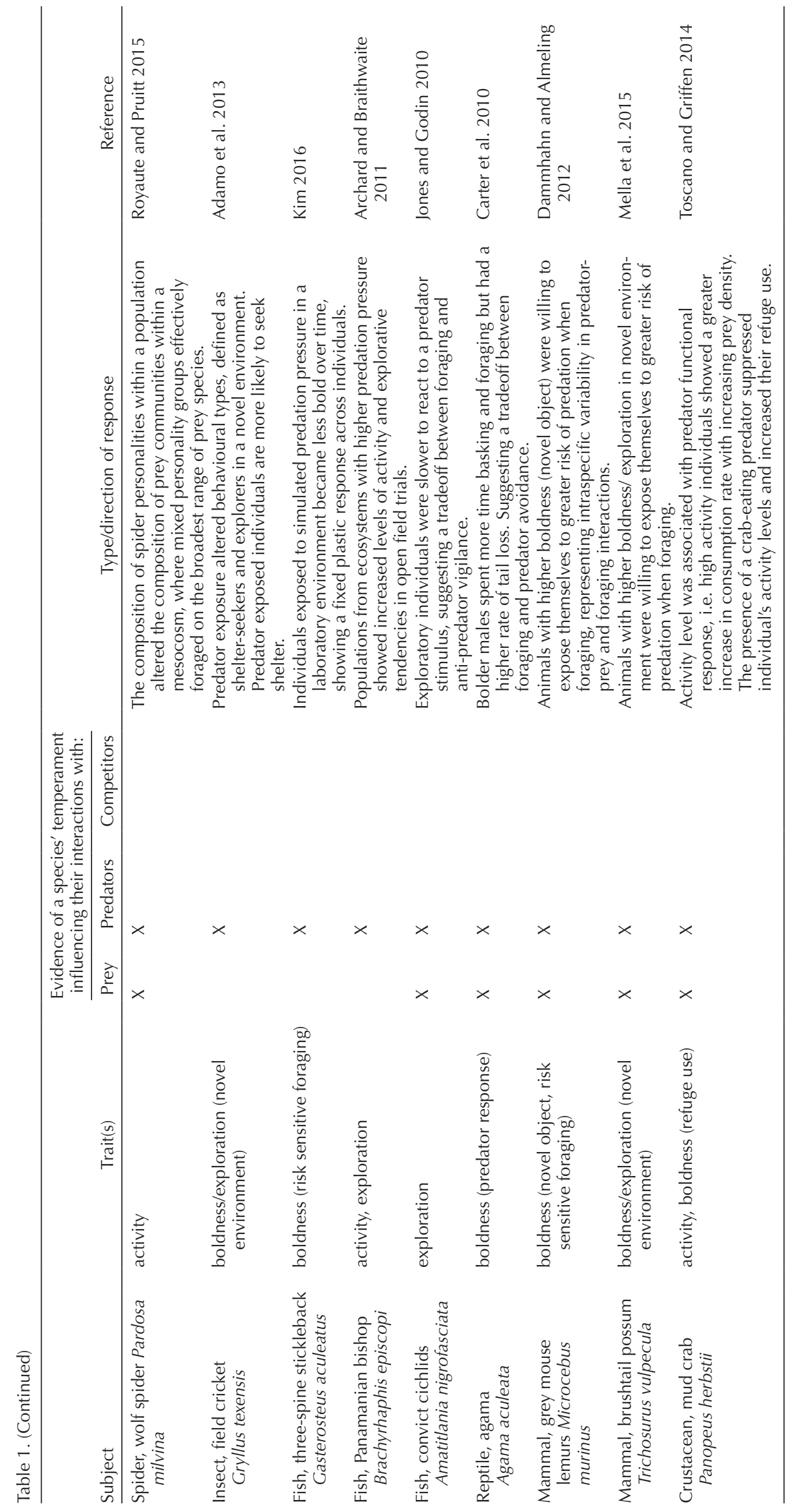

EV-6 


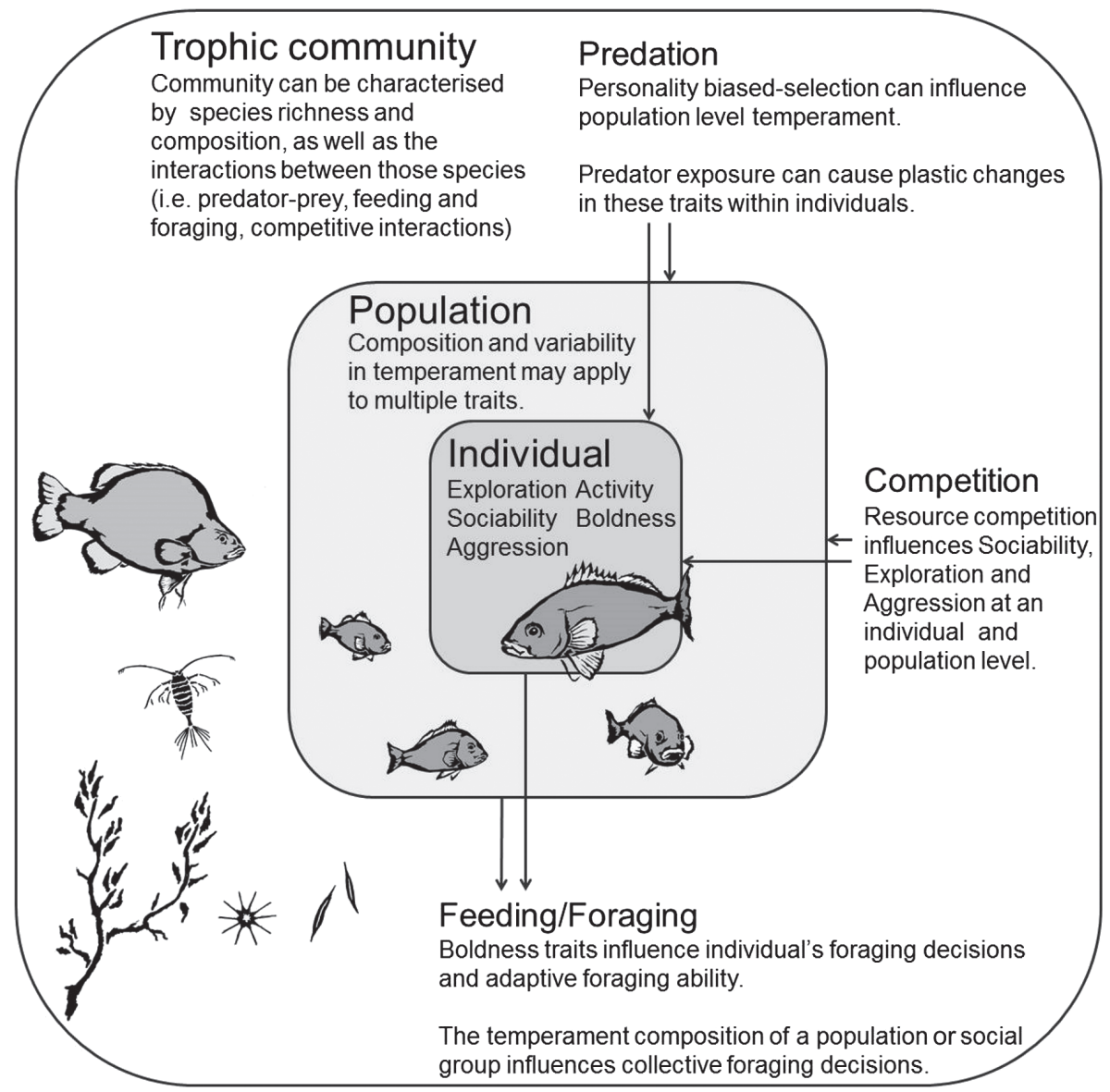

Figure 1. Individual temperament traits within a population and community context.

(Ferrari et al. 2014). An implication of this is that individuals with different temperament traits are more able to adapt and change their foraging behaviours than others. This has been observed in great tits, where more exploratory individuals were better able to cope with the loss of a food source by switching to spatially different sources (van Overveld and Matthysen 2010). These examples suggest that adaptive/ non-adaptive foraging is itself an intraspecific behavioural axis. How individual and population ATB interact, particularly how the presence of more adaptive individual foragers influences the collective ability of populations to adapt to change, is an important question for future research.

\section{Predator-prey interactions: the prey's perspective}

An animal's temperament influences the outcome of individual interactions with their predators. Risk-taking tendencies, i.e. boldness traits, are often conflated with predator response behaviours, as the risk of being predated is highly relevant to an animal's risk-taking tendencies in a natural setting. A common method of testing boldness is through predator exposure or fright stimulus assays (Wilson and Godin 2009, Chapman et al. 2010), such that individual boldness differences within populations can imply that there are intraspecific differences that effect predator-prey interactions (Dosmann and Mateo 2014). Furthermore, studies have shown that temperament traits can predict an individual's susceptibility to predation. For example, in a beetle Tenebrio molitor, boldness was associated with greater susceptibility to aerial predation (Krams et al. 2013), while more exploratory lemon damselfish Pomacentrus moluccensis had higher survival rates when exposed to a predator (White et al. 2013). In situations where both predator and prey have variation in temperament, the outcome of predator and prey interaction is jointly determined by both behavioural types (DiRienzo et al. 2013, Sweeney et al. 2013). For black turban snails Chlorostoma funebralis and their predator, ocher sea stars Pisaster ochraceus, more active predators favoured snails with greater predator avoidance behaviours whereas less active predators favoured snails with low predator avoidance behaviour (Pruitt et al. 2012b). Temperament can have fitness consequences for individuals relating to predation and is a source of intraspecific variability influencing predator-prey interactions, potentially leading to changes in prey foraging behaviour and altering predatorprey functional responses.

Temperament traits of prey species are also associated with phenotypic responses to predation. Sub-lethal interactions with predators may lead to shifts in individual's behavioural response traits. For example experiments in a field cricket Gryllus texensis showed that individuals demonstrated increased shelter-seeking behaviour post-exposure (Adamo et al. 2013). Similarly in three-spined sticklebacks, individuals had behavioural types with reduced aggression following predator exposure (Bell and Sih 2007). These changes in behavioural responses may represent shifts in phenotype in response to environmental factors, e.g. past predator 
exposure, which is potentially a form of plasticity distinct from flexible, context-specific behavioural responses.

Temperament traits of prey populations have been shown to be associated with the level of predation pressure, which suggests that temperament does not necessarily inhibit responses to predation pressure and may mitigate the effects of predator interactions (Brydges et al. 2008, Harris et al. 2010, Archard and Braithwaite 2011). A long term study of Eurasian perch Perca fluviatilis showed that, over six years, increases in predation pressure were associated with reduced boldness levels of juvenile fish (Magnhagen et al. 2012). The mechanisms for these population-level responses appear to be a combination of differences in selective pressure associated with behavioural types and plastic personality responses to sub-lethal interactions. Bell and Sih's (2007) predator exposure and predation survival experiment on three-spined sticklebacks showed that selection and plastic responses combined to influence the population's behavioural characteristics. Non-aggressive and bold individuals had the highest rates of mortality, whereas non-lethal predator exposure induced a decrease in aggression, but not boldness. This suggests that temperament responses to predation potentially have a significant effect on the characteristics of that predator-prey interaction.

Studies that have incorporated multiple trophic interactions provide further evidence that temperament is a source of intraspecific variability influencing trophic interactions and that this can mitigate the cascading effects of predation pressure on communities. An example is the relationship between food and fear in meso-predators, which experience the dual ecological pressures of foraging and dealing with predation pressure (McArthur et al. 2014). There is increasing evidence that there may be a tradeoff between anti-predator behaviours/predator vigilance and foraging decisions, and temperament plays a role in this (Stamps 2007). For example, in the agama lizard Agama aculeata, exploratory individuals that spent more time foraging had a higher rate of tail loss, suggesting greater susceptibility to predation (Carter et al. 2010). Risk-sensitive foraging as a boldness trait, which measures an animal's willingness to forage in areas of high-predation risk, explores this tradeoff as it is inherently related to both foraging and predator interactions. For example, both grey mouse lemurs Microcebus murinus (Dammhahn and Almeling 2012) and brushtail possums Trichosurus vulpecula (Mella et al. 2015) that are bolder in a novel object test were willing to expose themselves to a greater risk of predation when feeding. This has been characterised as a behavioural mechanism to mitigate the effects of predation through differences in foraging effort (Elvidge et al. 2014). Furthermore, this demonstrates how temperament traits may influence how perturbations propagate through food webs, by dampening the negative impacts of strong predator-prey interactions.

\section{Competition and temperament}

Temperament has been shown to drive competitive interactions both within and between species (Blight et al. 2016, Lichtenstein et al. 2016), and may facilitate adaptive responses under competition. Aggression and exploration are common traits studied in this context. For instance, explor- atory behaviour was positively correlated with competitive success in great tits (Cole and Quinn 2012), but negatively correlated in the zebra finch (David et al. 2011). In a mammal, Merriam's kangaroo rat Dipodomys merriami, interspecific competition was positively correlated with increased aggression (Dochtermann et al. 2012). The fitness of eastern bluebirds Sialia sialis under high interspecific competition favoured highly aggressive or non-aggressive behavioural types over intermediate types (Harris and Siefferman 2014). Intraspecific behavioural differences potentially reduce the strength of interspecific competitive interactions and may mitigate the negative effects of competition on community stability (Bolnick et al. 2011). Understanding competition as a potential driver of intraspecific behavioural differences and the nature of individual behavioural responses to competition is crucial to the integration of behavioural and community ecology.

\section{Moving to the whole food web}

Several characteristics of temperament traits may have broad-scale effects on the structural and dynamic features of food webs. Temperament traits are characterised by partial consistency, where meta-analysis has shown the mean repeatability across behavioural traits to be approximately 0.37 (where $0=$ no repeatability, $1=$ absolute repeatability, Bell et al. 2009). The framework of behavioural reaction norms characterises behavioural traits according to both their repeatable and plastic components, which are both potentially subject to ecological pressures (Dingemanse et al. 2010). Additionally, behavioural traits may show phenotypic plasticity, via changes to the mechanisms underlying repeatable behavioural traits due to environmental effects (e.g. hormonal responses), such as early life experience with food limitation or non-lethal exposure with a predator (McGhee and Travis 2011, Adamo et al. 2013). Considering each of these characteristics of behavioural traits, temperament may influence food-web interactions through intraspecific variability, but also by affecting adaptive responses in populations through temperament-biased selection and in individuals through behavioural and phenotypic plasticity. Incorporating this is crucial to our understanding of the structural and dynamic properties of real world food webs and the role of biodiversity within those communities (Fig. 2).

\section{Community modelling techniques}

Studies of ecological communities often use network analysis approaches to quantify stability at a community level. Analytical approaches can be broadly divided into two approaches (Thompson et al. 2012b). Firstly, structural approaches which describe the topology of networks in terms of the species present and the architecture of their interactions (Dunne 2006, Allesina et al. 2008). Principle inputs into this analysis include the number of species interacting and connectance, i.e. the links between those species (Brose et al. 2006). Emergent variables from structural analysis can infer greater stability, persistence or robustness in a food web, such as the distribution of links among species, the mean trophic level among consumer species, the mean and distribution of interaction strengths and the degree of 


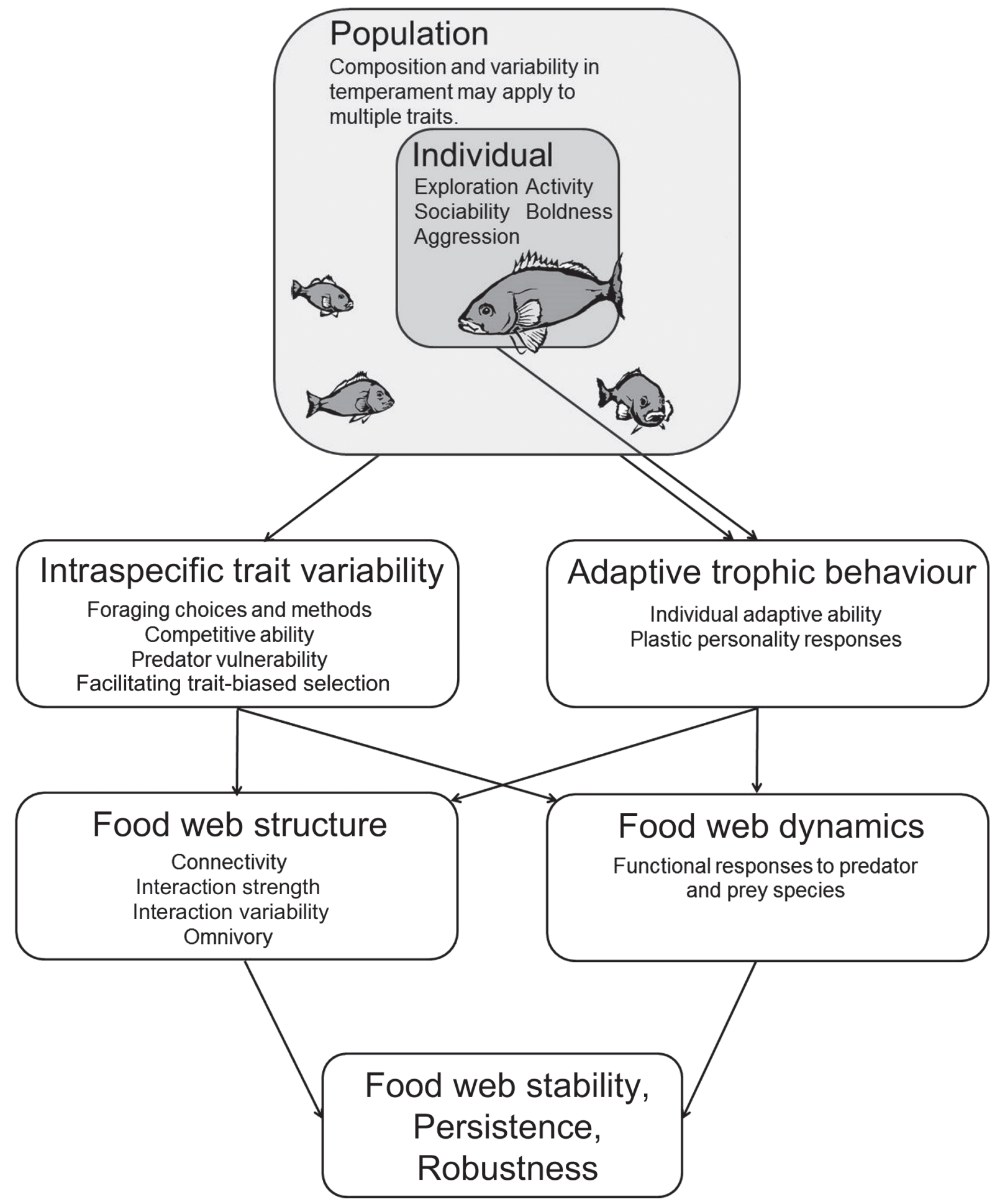

Figure 2. Linking temperament to food web effects.

omnivory, particularly in top-predator species (Dunne et al. 2002, Martinez et al. 2006, Gross et al. 2009, Allesina and Tang 2012).

The second approach analyses the dynamics of food webs to predict the temporal variability in the abundance or biomass of constituent species or the likelihood that species (or nodes) and the links between nodes will disappear from the food web. Generally this approach employs general population dynamic equations of the form (Valdovinos et al. 2010):

$\frac{\mathrm{dN}_{j}}{\mathrm{~d} t}=\mathrm{r}_{j} \mathrm{~N}_{j}+\sum_{i \in \mathrm{R}_{j}} \mathrm{e}_{i j} \mathrm{~g}_{i j} \mathrm{~N}_{j}-\sum_{k \in \mathrm{C}_{j}} \mathrm{~g}_{j k} \mathrm{~N}_{k}$

For a general species, $j$, inputs are their abundance, biomass or density $\left(\mathrm{N}_{j}\right)$, their intrinsic growth rate $\left(\mathrm{r}_{j}\right)$, their functional 
response $\left(\mathrm{g}_{i j}\right)$ and conversion efficiency $\left(\mathrm{e}_{i j}\right)$ of prey species $i$, and their functional response to a predator species, $k\left(\mathrm{~g}_{j k}\right)$, of size $\mathrm{N}_{k}$. By altering the terms of this equation the theoretical effects of ecological characteristics on food-web stability can be analysed, such as allometric scaling (Brose et al. 2006) and compartmentalisation (Stouffer and Bascompte 2011). These approaches allow us to investigate the community characteristics that influence the stability, persistence and robustness of food webs, including structural patterns (Martinez et al. 2006). For example the effects of adaptive behaviour in prey species may be analysed, in part, by modifying the functional response factors, $\mathrm{g}_{j k}$, to predator $k$, which can have stabilising effects (Kondoh 2007). As the modelling of the repeatable and plastic components of behaviour are rapidly progressing (Dingemanse et al. 2010), this is a unique opportunity to explore the effects on food webs of adaptive responses stemming from plasticity in behavioural responses and phenotypes. Where introducing these elements enhances stability in larger, more complex communities, systemic selection would promote greater biodiversity in ecosystems (Borrelli et al. 2015). This is a mechanism through which intraspecific behavioural variability may enable complex species-rich food webs to persist and is a means to answering the biodiversity paradox.

\section{Temperament as a source of intraspecific trait variability}

The structure and topography of food webs is altered by the presence of intraspecific trait variability. This is because interactions between individuals are the interface where foodweb interactions actually occur (Thompson et al. 2012b). In addition to the within-population effects outlined above, Bolnick et al. (2011) proposes multiple mechanisms through which intraspecific trait variability alters the strength and distribution of trophic interactions. For example, where a trait is non-linearly related to an interaction (Jensen's inequality; Ruel and Ayres 1999), mean interaction strength and the functional response to predator/prey species are altered. Similarly the presence of trait variation that influences trophic interactions will tend to decrease the strength of interactions but increase node degree, i.e. the number of links to a node/species, which are thought to increase stability and robustness in food webs (Bolnick et al. 2011). Pruitt et al. (2012a) manipulated the composition of aggressive temperaments in spider colonies (Anelosimus studiosus), where aggressive, docile and mixed colonies each had differing interactions with their local insect community. This also had indirect effects on interspecific interactions throughout their community, showing that both the mean and variance of temperament traits in one species can have wide ranging effects at this level.

Similarly, trait-biased selection can drive rapid population level shifts in phenotype where those traits are at least partially heritable. Where this occurs under predation for example, the selection is likely to alter the species' functional response to that predator (Bell and Sih 2007, Bolnick et al. 2011). Moya-Larano (2011) has shown how trait variability (in terms of growth rates) influences the structural components of model food webs by decreasing predator specialisation, resulting in increased connectance, omnivory and variation in interaction strengths and an overall decrease in interaction strengths (Moya-Larano 2011). These changes suggest that intraspecific differences have a positive effect on food-web stability and persistence.

Trophic cascades are an example where intraspecific variation, particularly in behavioural traits, may act as a buffer against changes to food-web structure and function. Trophic cascades occur when changes in abundance of one species, often a top predator, has alternating positive and negative effects moving down food chains, leading to significantly altered community structure and function (Frank et al. 2005). Within population behavioural differences, as a source of intraspecific variability affecting trophic interactions, are likely to alter the magnitude of trophic cascades. Harmon et al. (2009) showed that within-population differences in feeding behaviour of three-spined sticklebacks had community-level effects in mesocosms. Treatments showed that the structure of prey communities differed between systems with generalist-feeding fish versus systems that contained specialized-feeding types (both benthic and limnetic feeders). Keiser et al. (2015) similarly used mesocosms to show how the composition of behavioural types in spider populations influenced the extent of leaf damage in plants via indirect effects on their herbivores. These studies suggest that behavioural traits should be a key focus as we explore the community effects of intraspecific variability, through mechanisms including altered predator/prey functional responses and mean interaction strengths across food-webs (Bolnick et al. 2011).

A significant body of evidence shows that temperament traits are a source of intraspecific variation in foodweb interactions, including in susceptibility to predation (Adamo et al. 2013) and foraging choices/ methods (David et al. 2012, Rockwell et al. 2012). There is less known about the relative effects of behaviour and other individual-level traits, such as metabolic and physiological differences, in food-webs (Moya-Larano 2011). Although evidence points to temperament altering interactions in a way that is likely to enhance stability and persistence in food webs, further research is required to establish whether the effects of temperament on trophic interactions are sufficiently large to induce structural and dynamic differences at the community level.

\section{Temperament's role in adaptive trophic behaviour}

Adaptive trophic behaviour influences both the structural and dynamic characteristics of food webs (Valdovinos et al. 2010). ATB includes both adaptive foraging, i.e. adjusting foraging efforts to factors such as changing prey abundance, and the ability to respond adaptively to the animals that consume them, i.e. predator response. Various approaches have been used to incorporate ATB into dynamic food-web modelling, generally by altering the functional responses of species to prey and predator species to a non-linear adaptive response (Valdovinos et al. 2010). This work generally shows that the presence of adaptive behaviour alters the community responses to environmental perturbations, enhancing plasticity in the food-web structure and producing more permanent, persistent food webs (Uchida et al. 2007, Heckmann et al. 2012). The effects of adaptive behaviour on food-web structure as predicted by models tend to show higher numbers of species and increased trophic levels (Guill 
and Drossel 2008), suggesting adaptive behaviour is an important characteristic within ecosystems underpinning biodiversity.

The relationship between ATB and temperament is complex, where rigid behavioural types can be seen as inhibiting an animal's optimum behavioural response to ecological conditions, potentially negatively influencing individual fitness and inhibiting ATB (Conrad et al. 2011, Sih et al. 2012). Nonetheless, temperament traits are only partially repeatable and the plastic component of behavioural reactions still allows individuals to adaptively respond to environmental stimuli (Dingemanse et al. 2010). For example, in the crab Panopeus herbstii, their functional response to a prey species - the mussel Brachidontes exustus - was related to their individual activity level, but this functional response changed in the presence of a crab predator independently of the effects of activity (Toscano and Griffen 2014). Furthermore, experimental studies suggest that there may be ontogenic effects on temperament traits due to various ecological pressures, including diet variability (Chapman et al. 2010), food resource competition (McGhee and Travis 2011) and predator exposure (Adamo et al. 2013). This requires further exploration of the how consistency in temperament traits interact with behavioural plasticity, particularly understanding the dual roles of genetics and environmental conditions in determining behavioural types (Brown et al. 2007, van Oers and Mueller 2010). This will allow behavioural adaptability to be more effectively incorporated into our understanding of community stability.

\section{Conclusions}

Both behavioural ecology and community ecology are rapidly evolving. In behavioural ecology there is increasing interest in the role of individual temperament as a process underpinning natural selection and evolution (Réale et al. 2007, Wolf and Weissing 2012). In food-web ecology, increasingly sophisticated modelling approaches are seeking to understand the processes that underpin trophic structure, including through the use of individualbased models (Thompson et al. 2012b). However there are remarkably few studies at the nexus of these two disciplines, where many key questions remain unresolved. Viewing temperament studies in the light of community analysis, it is clear that there is potential for animal behaviour to influence food-web topology and dynamics, including apparently stabilizing effects. These stability effects are critical to how we understand complexity and biodiversity within natural food webs, and can contribute to resolving the biodiversity paradox. The relationship between temperament and stability is also critical to our understating of how individual behavioural differences develop in populations, particularly if temperament is a positive force on community stability simply as an emergent property of a population's competitive and predator-prey interactions. This would represent an ecological pressure encouraging the development and maintenance of individual behavioural differences and potentially account for the apparent non-adaptive nature of certain individual behavioural types (Borrelli et al. 2015).
Although there is significant evidence of an interactive relationship between an animal's trophic environment and animal temperament, it is unlikely that these responses are linear due to the multiplicity of factors that can influence temperament traits, e.g. abiotic effects (Frost et al. 2013), social effects (Webster and Ward 2011, Jolles et al. 2013) and mating behaviours (Magnhagen et al. 2014). Understanding and incorporating the non-linear nature of behavioural traits into food-web dynamics will be a significant challenge to community ecologists in the future. Nonetheless, as we continue to explore the dynamics of individual behaviour and of communities, it is clear that they are mutually relevant and their integration is a critical future step.

\section{Future research questions}

1) Animals can show intraspecific differences in their ability to adapt to changing food sources. How does the mean and variance of adaptive behavioural types within a population influence their collective adaptive ability?

2) How do multiple interactions, such as competition and predation, combine to influence temperament in populations? Are their influences non-independent?

3) How significant are the effects of intraspecific behavioural variability on food-web structure and trophic cascades relative to other intraspecific traits, such as morphological or physiological traits? How do behavioural and morphological/ physiological effects on food webs interact?

4) How does the combination of plasticity and repeatability in temperament traits influence the functional response of a species with a predator or prey species, and what are the relative influences of plasticity and repeatability on predator-prey interactions?

5) How do the dual effects of temperament in constraining and facilitating adaptive responses to ecological pressures influence dynamic food-web modelling?

Acknowledgements - We thank all who contributed to this research, particularly within the Monash Behavioural Ecology and Freshwater Ecology Research Groups.

Funding - We received financial support from the Australian Research Council (DP120103010) and NM received additional support from the Holsworth Wildlife Endowment and the Great Artesian Basin Coordinating Committee.

Statement of authorship - NM wrote the main manuscript and prepared all figures and tables. All authors contributed to the conception of the ideas described therein, and all authors edited and reviewed the manuscript.

\section{References}

Adamo, S. A. et al. 2013. The behavioural effects of predatorinduced stress responses in the cricket (Gryllus texensis): the upside of the stress response. - J. Exp. Biol. 216: 4608-4614.

Akihiko, M. and Michio, K. 2014. Stability of competitionantagonism-mutualism hybrid community and the role of community network structure. - J. Theor. Biol. 360: 54-58.

Allesina, S. and Tang, S. 2012. Stability criteria for complex ecosystems. - Nature 483: 205-208.

Allesina, S. et al. 2008. A general model for food web structure. - Science 320: 658-661. 
Ang, T. Z. and Manica, A. 2010. Aggression, segregation and stability in a dominance hierarchy. - Proc. R. Soc. B 277: 1337-1343.

Araujo, M. S. et al. 2011. The ecological causes of individual specialisation. - Ecol. Lett. 14: 948-958.

Archard, G. A. and Braithwaite, V. A. 2011. Increased exposure to predators increases both exploration and activity level in Brachyrhaphis episcopi. - J. Fish Biol. 78: 593-601.

Baiser, B. et al. 2010. Connectance determines invasion success via trophic interactions in model food webs. - Oikos 119: 1970-1976.

Bell, A. M. and Sih, A. 2007. Exposure to predation generates personality in threespined sticklebacks (Gasterosteus aculeatus). - Ecol. Lett. 10: 828-834.

Bell, A. M. et al. 2009. The repeatability of behaviour: a metaanalysis. - Anim. Behav. 77: 771-783.

Bergmüller, R. and Taborsky, M. 2010. Animal personality due to social niche specialisation. - Trends Ecol. Evol. 25: 504-511.

Binzer, A. et al. 2012. The dynamics of food chains under climate change and nutrient enrichment. - Phil. Trans. R. Soc. B 367: $2935-2944$.

Blight, O. et al. 2016. A proactive-reactive syndrome affects group success in an ant species. - Behav. Ecol. 27: 118-125.

Bolnick, D. I. et al. 2011. Why intraspecific trait variation matters in community ecology. - Trends Ecol. Evol. 26: $183-192$.

Borrelli, J. J. et al. 2015. Selection on stability across ecological scales. - Trends Ecol. Evol. 30: 417-425.

Brose, U. et al. 2006. Allometric scaling enhances stability in complex food webs. - Ecol. Lett. 9: 1228-1236.

Brown, C. et al. 2007. Heritable and experiential effects on boldness in a tropical poeciliid. - Behav. Ecol. Sociobiol. 62: 237-243.

Brydges, N. M. et al. 2008. Habitat stability and predation pressure affect temperament behaviours in populations of three-spined sticklebacks. - J. Anim. Ecol. 77: 229-235.

Careau, V. et al. 2008. Energy metabolism and animal personality. - Oikos 117: 641-653.

Carere, C. et al. 2005. Epigenetic effects on personality traits: early food provisioning and sibling competition. - Behaviour 142: 1329-1355.

Carter, A. J. et al. 2010. Agamas exhibit behavioral syndromes: bolder males bask and feed more but may suffer higher predation. - Behav. Ecol. 21: 655-661.

Chapman, B. B. et al. 2010. Unpredictability in food supply during early life influences boldness in fish. - Behav. Ecol. 21 : 501-506.

Clark, J. S. et al. 2007. Resolving the biodiversity paradox. - Ecol. Lett. 10: 647-659.

Cole, E. F. and Quinn, J. L. 2012. Personality and problem-solving performance explain competitive ability in the wild. - Proc. R. Soc. B 279: 1168-1175.

Conrad, J. L. et al. 2011. Behavioural syndromes in fishes: a review with implications for ecology and fisheries management. - J. Fish Biol. 78: 395-435.

Dammhahn, M. and Almeling, L. 2012. Is risk taking during foraging a personality trait? A field test for cross-context consistency in boldness. - Anim. Behav. 84: 1131-1139.

David, M. et al. 2012. Personality and body condition have additive effects on motivation to feed in Zebra Finches Taeniopygia guttata. - Ibis 154: 372-378.

David, M. et al. 2011. Personality affects zebra finch feeding success in a producer-scrounger game. - Anim. Behav. 82: 61-67.

Dingemanse, N. J. et al. 2010. Behavioural reaction norms: animal personality meets individual plasticity. - Trends Ecol. Evol. 25: 81-89.
DiRienzo, N. et al. 2013. The combined behavioural tendencies of predator and prey mediate the outcome of their interaction. - Anim. Behav. 86: 317-322.

Dochtermann, N. A. et al. 2012. The roles of competition and environmental heterogeneity in the maintenance of behavioral variation and covariation. - Ecology 93: 1330-1339.

Dosmann, A. and Mateo, J. M. 2014. Food, sex and predators: animal personality persists with multidimensional plasticity across complex environments. - Anim. Behav. 90: 109-116.

Dunne, J. A. 2006. The network structure of food webs. - In: Pascual, M. and Dunne, J. A. (eds), Ecological networks: linking structure to dynamics in food webs. Oxford Univ. Press, pp. 27-86.

Dunne, J. A. et al. 2002. Network structure and biodiversity loss in food webs: robustness increases with connectance. - Ecol. Lett. 5: 558-567.

Elvidge, C. K. et al. 2014. Compensatory foraging in Trinidadian guppies: effects of acute and chronic predation threats. - Curr. Zool. 60: 323-332.

Ferrari, S. et al. 2014. First links between self-feeding behaviour and personality traits in European seabass, Dicentrarchus labrax. - Appl. Anim. Behav. Sci. 161: 131-141.

Frank, K. T. et al. 2005. Trophic cascades in a formerly cod-dominated ecosystem. - Science 308: 1621-1623.

Frost, A. J. et al. 2013. Environmental change alters personality in the rainbow trout, Oncorhynchus mykiss. - Anim. Behav. 85: 1199-1207.

Gross, T. et al. 2009. Generalized models reveal stabilizing factors in food webs. - Science 325: 747-750.

Guill, C. and Drossel, B. 2008. Emergence of complexity in evolving niche-model food webs. - J. Theor. Biol. 251: $108-120$.

Harmon, L. J. et al. 2009. Evolutionary diversification in stickleback affects ecosystem functioning. - Nature 458: 1167-1170.

Harris, M. R. and Siefferman, L. 2014. Interspecific competition influences fitness benefits of assortative mating for territorial aggression in eastern bluebirds (Sialia sialis). - PloS One 9: e88668.

Harris, S. et al. 2010. Picking personalities apart: estimating the influence of predation, sex and body size on boldness in the guppy Poecilia reticulata. - Oikos 119: 1711-1718.

Heckmann, L. et al. 2012. Interactive effects of body-size structure and adaptive foraging on food-web stability. - Ecol. Lett. 15: 243-250.

Ho, S. S. et al. 2011. Comparing food-web impacts of a native invertebrate and an invasive fish as predators in small floodplain wetlands. - Mar. Freshwater Res. 62: 372-382.

Hubbell, S. P. 2006. Neutral theory and the evolution of ecological equivalence. - Ecology 87: 1387-1398.

Jolles, J. W. et al. 2013. Dominance, pair bonds and boldness determine social-foraging tactics in rooks, Corvus frugilegus. - Anim. Behav. 85: 1261-1269.

Jolles, J. W. et al. 2016. Food intake rates of inactive fish are positively linked to boldness in three-spined sticklebacks Gasterosteus aculeatus. - J. Fish Biol. 88: 1661-1668.

Jones, K. A. and Godin, J-G. J. 2010. Are fast explorers slow reactors? Linking personality type and anti-predator behaviour. - Proc. R. Soc. B 277: 625-632.

Keiser, C. N. and Pruitt, J. N. 2014. Personality composition is more important than group size in determining collective foraging behaviour in the wild. - Proc. R. Soc. B 281: 20141424.

Keiser, C. N. et al. 2015. Individual differences in predators but not producers mediate the magnitude of a trophic cascade. - Arthropod-Plant Interact. 9: 225-232. 
Kim, S. Y. 2016. Fixed behavioural plasticity in response to predation risk in the three-spined stickleback. - Anim. Behav. 112: 147-152.

Kobler, A. et al. 2009. Coexistence of behavioural types in an aquatic top predator: a response to resource limitation? - Oecologia 161: 837-847.

Kondoh, M. 2007. Anti-predator defence and the complexitystability relationship of food webs. - Proc. R. Soc. B 274: 1617-1624.

Krams, I. et al. 2013. Predation selects for low resting metabolic rate and consistent individual differences in anti-predator behavior in a beetle. - Acta Ethol. 16: 163-172.

Kurvers, R. H. J. M. et al. 2012a. No evidence for negative frequency-dependent feeding performance in relation to personality. - Behav. Ecol. 23: 51-57.

Kurvers, R. H. J. M. et al. 2012b. Boldness affects foraging decisions in barnacle geese: an experimental approach. - Behav. Ecol. 23: 1155-1161.

Laskowski, K. L. and Pruitt, J. N. 2014. Evidence of social niche construction: persistent and repeated social interactions generate stronger personalities in a social spider. - Proc. R. Soc. B 281: 20133166 .

Lichtenstein, J. L. L. et al. 2016. Intraspecific variation in collective behaviors drives interspecific contests in acorn ants. - Behav. Ecol. 27: 553-559.

Magnhagen, C. et al. 2012. Boldness in two perch populations long-term differences and the effect of predation pressure. $-\mathrm{J}$. Anim. Ecol. 81: 1311-1318.

Magnhagen, C. et al. 2014. Context consistency and seasonal variation in boldness of male two-spotted gobies. - PloS One 9: e93354.

Martinez, N. D. et al. 2006. Diversity, complexity and persistence in large model ecosystems. - In: Pascual, M. and Dunne, J. A. (eds), Ecological networks: linking structure to dynamics in food webs. Oxford Univ. Press, pp. 163-185.

May, R. M. 1972. Will a large complex system be stable. - Nature 238: 413-414.

McArthur, C. et al. 2014. The dilemma of foraging herbivores: dealing with food and fear. - Oecologia 176: 677-689.

McCann, K. S. 2000. The diversity-stability debate. - Nature 405: 228-233.

McDermott, D. R. et al. 2014. Boldness is influenced by sublethal interactions with predators and is associated with successful harem infiltration in Madagascar hissing cockroaches. - Behav. Ecol. Sociobiol. 68: 425-435.

McGhee, K. E. and Travis, J. 2011. Early food and social environment affect certain behaviours but not female choice or male dominance in bluefin killifish. - Anim. Behav. 82: $139-147$.

Mella, V. S. A. et al. 2015. Personality affects the foraging response of a mammalian herbivore to the dual costs of food and fear. - Oecologia 177: 293-303.

Michelena, P. et al.. 2010. Personality and collective decisionmaking in foraging herbivores. - Proc. R. Soc. B 277: 1093-1099.

Milo, R. et al. 2002. Network motifs: simple building blocks of complex networks. - Science 298: 824-827.

Modlmeier, A. P. et al. 2014a. Persistent social interactions beget more pronounced personalities in a desert-dwelling social spider. - Biol. Lett. 10: 20140419.

Modlmeier, A. P. et al. 2014b. Species-specific influence of group composition on collective behaviors in ants. - Behav. Ecol. Sociobiol. 68: 1929-1937.

Montiglio, P.-O. et al. 2013. Social niche specialization under constraints: personality, social interactions and environmental heterogeneity. - Phil. Trans. R. Soc. B 368: 2 0120343 .
Moya-Larano, J. 2011. Genetic variation, predator-prey interactions and food web structure. - Phil. Trans. R. Soc. B 366: 1425-1437.

Nannini, M. A. et al. 2012. Do behavioral syndromes affect foraging strategy and risk-taking in a juvenile fish predator? - Trans. Am. Fish. Soc. 141: 26-33.

Niemela, P. T. et al. 2012. Predator-induced changes in the boldness of naive field crickets, Gryllus integer, depends on behavioural type. - Anim. Behav. 84: 129-135.

Oudman, T. et al. 2016. Diet preferences as the cause of individual differences rather than the consequence. - J. Anim. Ecol. 85: 1378-1388.

Patrick, S. C. and Weimerskirch, H. 2014. Personality, foraging and fitness consequences in a long lived seabird. - PloS One 9: e87269.

Pruitt, J. N. et al. 2012a. Behavioural trait variants in a habitatforming species dictate the nature of its interactions with and among heterospecifics. - Funct. Ecol. 26: 29-36.

Pruitt, J. N. et al. 2012b. Behavioral types of predator and prey jointly determine prey survival: potential implications for the maintenance of within-species behavioral variation. - Am. Nat. 179: 217-227.

Ravigne, V. et al. 2009. Live where you thrive: joint evolution of habitat choice and local adaptation facilitates specialization and promotes diversity. - Am. Nat. 174: E141-E169.

Réale, D. and Festa-Bianchet, M. 2003. Predator-induced natural selection on temperament in bighorn ewes. - Anim. Behav. 65: 463-470.

Réale, D. et al. 2007. Integrating animal temperament within ecology and evolution. - Biol. Rev. 82: 291-318.

Rockwell, C. et al. 2012. Bolder, older, and selective: factors of individual-specific foraging behaviors in Steller's jays. - Behav. Ecol. 23: 676-683.

Royaute, R. and Pruitt, J. N. 2015. Varying predator personalities generates contrasting prey communities in an agroecosystem. - Ecology 96: 2902-2911.

Ruel, J. J. and Ayres, M. P. 1999. Jensen's inequality predicts effects of environmental variation. - Trends Ecol. Evol. 14: 361-366.

Sih, A. et al. 2012. Ecological implications of behavioural syndromes. - Ecol. Lett. 15: 278-289.

Stamps, J. A. 2007. Growth-mortality tradeoffs and 'personality traits' in animals. - Ecol. Lett. 10: 355-363.

Stouffer, D. B. and Bascompte, J. 2011. Compartmentalization increases food-web persistence. - Proc. Natl Acad. Sci. USA 108: 3648-3652.

Svanbäck, R. and Bolnick, D. I. 2007. Intraspecific competition drives increased resource use diversity within a natural population. - Proc. R. Soc. B 274: 839-844.

Sweeney, K. et al. 2013. Predator and prey activity levels jointly influence the outcome of long-term foraging bouts. - Behav. Ecol. 24: 1205-1210.

Tang, S. et al. 2014. Correlation between interaction strengths drives stability in large ecological networks. - Ecol. Lett. 17: 1094-1100.

Thompson, R. M. et al. 2012a. Food webs: reconciling the structure and function of biodiversity. - Trends Ecol. Evol. 27: 689-697.

Thompson, R. M. et al. 2012b. Freshwater food webs: towards a more fundamental understanding of biodiversity and community dynamics. - Freshwater Biol. 57: 1329-1341.

Toscano, B. J. and Griffen, B. D. 2014. Trait-mediated functional responses: predator behavioural type mediates prey consumption. - J. Anim. Ecol. 83: 1469-1477.

Uchida, S. et al. 2007. The structure of food webs with adaptive behaviour. - Ecol. Model. 206: 263-276. 
Valdovinos, F. S. et al. 2010. Consequences of adaptive behaviour for the structure and dynamics of food webs. - Ecol. Lett. 13: 1546-1559.

van Oers, K. and Mueller, J. C. 2010. Evolutionary genomics of animal personality. - Proc. Natl Acad. Sci. USA 365: 3991-4000.

van Overveld, T. and Matthysen, E. 2010. Personality predicts spatial responses to food manipulations in free-ranging great tits (Parus major). - Biol. Lett. 6: 187-190.

Webster, M. M. and Ward, A. J. W. 2011. Personality and social context. - Biol. Rev. 86: 759-773.

Wenfeng, F. and Kazuhiro, T. 2014. Heterogeneity in ecological mutualistic networks dominantly determines community stability. - Sci. Rep. 4: 5912.
White, J. R. et al. 2013. A comparison of measures of boldness and their relationships to survival in young fish. - PloS One 8: e68900.

Wilson, A. D. M. and Godin, J. G. J. 2009. Boldness and behavioral syndromes in the bluegill sunfish, Lepomis macrochirus. - Behav. Ecol. 20: 231-237.

Wilson, A. J. et al. 2011. Integrating personality research and animal contest theory: aggressiveness in the green swordtail Xiphophorus helleri. - PloS One 6(11): e28024.

Wolf, M. and Weissing, F. J. 2012. Animal personalities: consequences for ecology and evolution. - Trends Ecol. Evol. 27: $452-461$. 